\title{
Genome-wide analysis of translation reveals a critical role for deleted in azoospermia-like (Dazl) at the oocyte-to-zygote transition
}

\author{
Jing Chen, ${ }^{1,2,3}$ Collin Melton, ${ }^{1,2,4,7}$ Nayoung Suh, ${ }^{1,2,4,7}$ Jeong Su Oh, ${ }^{1,2,3}$ Kathleen Horner, ${ }^{1,2,3}$ \\ Fang Xie, ${ }^{1,2,3}$ Claudio Sette, ${ }^{5,6}$ Robert Blelloch, ${ }^{1,2,4}$ and Marco Conti ${ }^{1,2,3,8}$ \\ ${ }^{1}$ Center for Reproductive Sciences, University of California at San Francisco, San Francisco, California 94143, USA; ${ }^{2}$ The Eli and \\ Edythe Broad Center of Regeneration Medicine and Stem Cell Research, University of California at San Francisco, San Francisco, \\ California 94143, USA; ${ }^{3}$ Department of Obstetrics, Gynecology, and Reproductive Sciences, University of California at San \\ Francisco, San Francisco, California 94143, USA; ${ }^{4}$ Department of Urology, University of California at San Francisco, San \\ Francisco, California 94143, USA; ${ }^{5}$ Department of Public Health, University of Rome Tor Vergata, Rome 00133, Italy; \\ ${ }^{6}$ Laboratory of Neuroembryology, Fondazione Santa Lucia, Rome 00143, Italy
}

Oocyte maturation, fertilization, and early embryonic development occur in the absence of gene transcription. Therefore, it is critical to understand at a global level the post-transcriptional events that are driving these transitions. Here we used a systems approach by combining polysome mRNA profiling and bioinformatics to identify RNA-binding motifs in mRNAs that either enter or exit the polysome pool during mouse oocyte maturation. Association of mRNA with the polysomes correlates with active translation. Using this strategy, we identified highly specific patterns of mRNA recruitment to the polysomes that are synchronized with the cell cycle. A large number of the mRNAs recovered with translating ribosomes contain motifs for the RNA-binding proteins DAZL (deleted in azoospermia-like) and CPEB (cytoplasmic polyadenylation element-binding protein). Although a Dazl role in early germ cell development is well established, no function has been described during oocyte-to-embryo transition. We demonstrate that CPEB1 regulates Dazl post-transcriptionally, and that DAZL is essential for meiotic maturation and embryonic cleavage. In the absence of DAZL synthesis, the meiotic spindle fails to form due to disorganization of meiotic microtubules. Therefore, Cpeb1 and Dazl function in a progressive, self-reinforcing pathway to promote oocyte maturation and early embryonic development.

[Keywords: meiosis; translation; oocyte; maternal mRNAs]

Supplemental material is available for this article.

Received January 4, 2011; revised version accepted February 22, 2011.

During development of the female gamete, control of gene expression is transferred from the nucleus to the cytoplasm (Seydoux and Braun 2006). Maternal mRNAs are actively transcribed throughout oocyte growth; however, de novo mRNA synthesis ceases during the final stages of oocyte maturation and early embryonic divisions. At these stages, previously synthesized maternal mRNAs are derepressed and translated according to a well-orchestrated program of recruitment to the polysomes and translational activation. Thus, cell cycle progression and genome reprogramming after fertilization rely exclusively on translation. Whereas some of the basic

\footnotetext{
${ }^{7}$ These authors contributed equally to this work.

${ }^{8}$ Corresponding author.

E-MAIL contim@obgyn.ucsf.edu; FAX (415) 502-7866.

Article is online at http://www.genesdev.org/cgi/doi/10.1101/gad.2028911.
}

mechanisms of translation repression and activation have been elucidated in model organisms, little is known of the processing of maternal mRNAs in mammals, including humans. In Drosophila, Caenorhabditis elegans, and Xenopus, mRNA translation plays critical roles in development: It is regulated in both time and space (Kimble and Crittenden 2007), and transport, storage, and translation of maternal mRNAs direct germline allocation and germline stem cell maintenance.

A comprehensive representation of all proteins translated at any given time during oocyte-to-zygote transition is critical for understanding the molecular basis of cell cycle control as well as the reprogramming of zygote and embryo genome activation. Proteomic approaches have been used to monitor protein synthesis in oocytes (Vitale et al. 2007; Zhang et al. 2009; Han et al. 2010; Wang et al. 2010), but the resolution achieved thus far provides 
insufficient insight into the regulation of maturation, as low-abundance proteins are difficult to quantitate by this approach. Although nucleic acid detection is quite sensitive, the strategy most frequently used involves the analysis of the transcriptome of oocytes and embryos (Latham et al. 2000; Oh et al. 2000; Evsikov et al. 2006; Su et al. 2007). This approach is useful to identify unstable mRNAs, but provides little information on protein synthesis because many maternal mRNAs in the oocyte are not translated. Here we used a different strategy to probe the pattern of protein expression in the oocyte by exploring the translation of maternal mRNAs globally.

Some of the basic mechanisms of translational regulation of maternal mRNAs during progression of the meiotic cell cycle have been elucidated in Xenopus oocytes (Richter 2007). Unmasking of dormant mRNAs and polyadenylation directed by cytoplasmic polyadenylation element (CPE) and its cognate binding protein (CPEB1) is thought to be a primary mechanism controlling translation (Radford et al. 2008). A combinatorial code of CPEs based on the properties of the $3^{\prime}$ untranslated region (UTR) of cyclin B1-B5 may account for the different timing of polyadenylation in prophase and metaphase I (MI) (Pique et al. 2008). Whether this arrangement of CPEs is sufficient to explain all of the waves of translation during different phases of meiosis is, however, controversial (Radford et al. 2008). CPEB-independent mechanisms likely contribute to generating temporal patterns of translation throughout maturation (Padmanabhan and Richter 2006; Arumugam et al. 2009). Moreover, regulated deadenylation provides an additional layer of control of translation in Xenopus oocytes (Belloc and Mendez 2008; Belloc et al. 2008).

Several additional RNA-binding proteins (RBPs) - the class of FBF (fem-3-binding factor)/PUF (Pumilio and FBF) RNA modulators being the most prominent-have been implicated in maintenance of stem cell identity and are required for mitotic divisions of germ cells in C. elegans and Drosophila. In these species, the family of GLD and FOG-1 proteins, a CPEB ortholog, is part of a complex regulatory network that controls other aspects of germ cell proliferation and differentiation, including meiosis (Kimble and Crittenden 2007). A role of the deleted in azoospermia (Daz) family of RBPs is also well established, as this family of genes is expressed predominantly in, and is essential for, primordial germ cell (PGC) development (Maines and Wasserman 1999; Houston and King 2000; Karashima et al. 2000), a function that is conserved in mammals (Reijo et al. 1995; Ruggiu et al. 1997). However, Dazl (Daz-like) expression persists during oocyte growth, but its role during these stages of gamete development is unknown. Although translational regulation of mRNA in mammalian oocytes was recognized early on (Huarte et al. 1992), how mRNA translation or destabilization is regulated at the egg-to-zygote transition in these species remains poorly understood (Oh et al. 2000; Schultz 2002; Evsikov et al. 2006).

Here we used genome-wide profiling of maternal mRNA recruitment to, or release from, the polysome to explore the pattern of translational regulations during oocyte maturation. In addition to providing insight into synthesis of proteins during maturation, we identified a novel mechanism that controls the late wave of translation in oocytes. We show that CPEB1 initially regulates the translation of DAZL, and that this RBP in turn regulates the translation of several downstream transcripts. These translational controls are essential for the oocyte-to-zygote transition.

\section{Results} Analysis of the pattern of $m R N A$ recruitment
to the polysomes during oocyte maturation

Polysomes and ribonucleoprotein particles (RNPs) from in vivo matured germinal vesicle (GV), MI, and metaphase II (MII) mouse oocytes were separated by density gradient centrifugation, and transcripts associated with the polysomes were further analyzed. By using microarray analysis of mRNAs recovered in the polysome fractions, we identified a highly organized, stage-dependent pattern of mRNA recruitment to the translating pool of ribosomes. Association of mRNA with the polysomes is an established approach to assess translation in vivo (Arava et al. 2003). Approximately 7600 transcripts are translated in maturing oocytes. Unsupervised clustering of the data revealed that transcript association with polysomes follows a stereotypical pattern during meiotic maturation (Supplemental Fig. S1). The hybridization data for 34 randomly chosen transcripts were confirmed by quantitative PCR (qPCR) on different sets of samples (Supplemental Figs. S2, S3). Given the transcriptional silencing of the fully grown oocyte (De La Fuente and Eppig 2001; Liu and Aoki 2002; De La Fuente et al. 2004), changes in transcript levels in the polysome fraction are due to transfer from or to the RNP fraction or destabilization but not de novo synthesis. Indeed, the translocation between the RNP and polysome compartments for representative transcripts was verified by qPCR (Supplemental Figs. S2, S3). Ccnb1 (Cyclin B1) mRNA translocation between compartments is associated with the accumulation of the CCNB1 protein (Supplemental Fig. S4). This association is verified with five other proteins known to be synthesized during maturation (MAGOH, WEE1B, b-CATENIN, SPINDLIN, and MOS) (Tay et al. 2000; Evsikov et al. 2006), confirming that this strategy correctly predicts protein synthesis during maturation.

Using this genome-wide analysis of translation, we identified three major classes of transcripts with distinct patterns of ribosome recruitment in GV and MII oocytes (false discovery rate $[\mathrm{FDR}]<5 \%, P<0.05$ ) (Fig. 1B,C). Although the majority of transcripts is constitutively on the polysomes (less than twofold change, class I, 4772 transcripts), one group of transcripts decreased (more than twofold, class II, 1519 transcripts) and another increased (more than twofold, class III, 1313 transcripts) in the polysome fraction during oocyte maturation.

Comparison of class II with available data sets (Su et al. 2007) of total transcripts in GV and MII oocytes reveals at least two different mechanisms of translational repression. Approximately 800 transcripts are unstable as total 


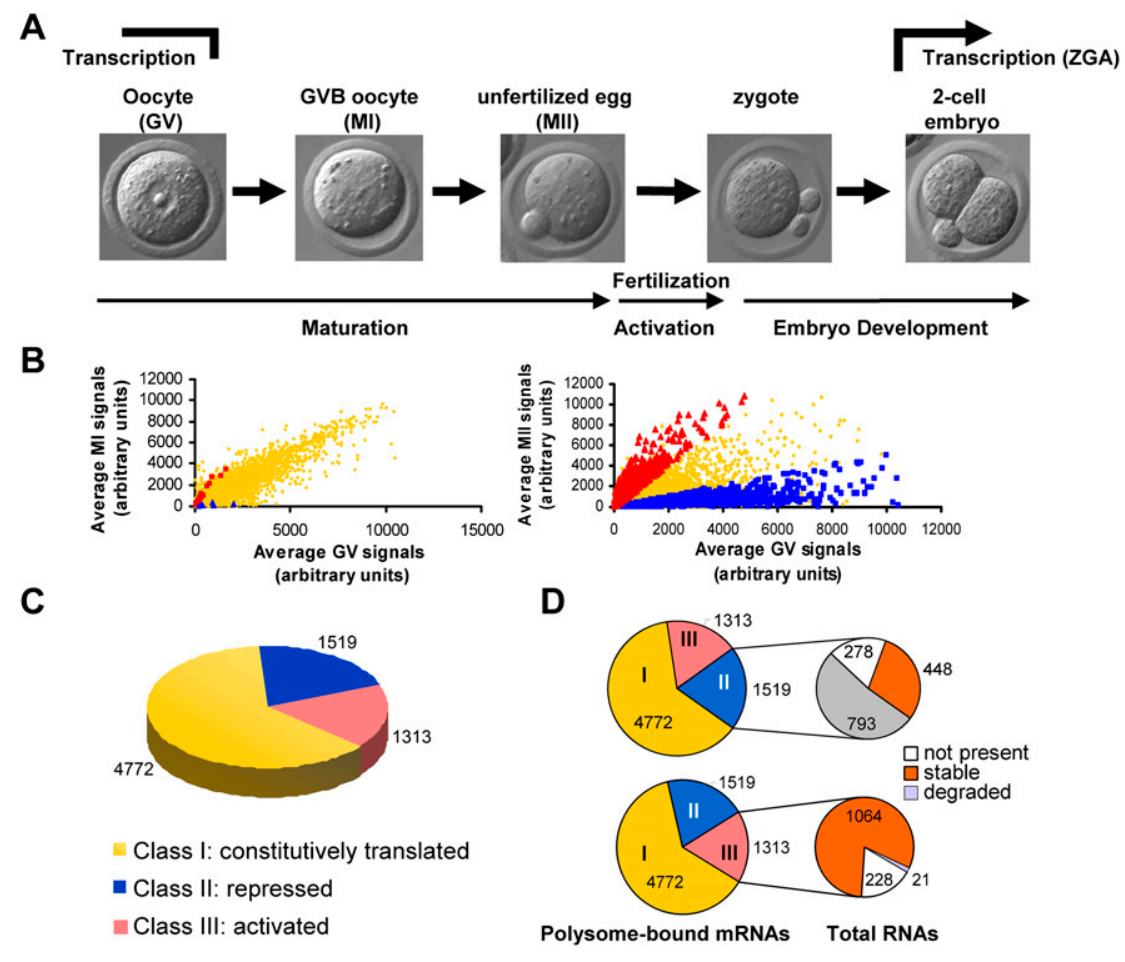

Figure 1. Genome-wide analysis of transcripts recovered from polyribosomes during oocyte maturation. (A) Schematic representation of the stages of oocyte maturation and early embryo development. Fully grown mouse oocytes are arrested in prophase I with prominent nucleus/nucleolus, termed GV. Within $3 \mathrm{~h}$ after $\mathrm{LH} / \mathrm{hCG}$ stimulation, oocytes re-enter the cell cycle with breakdown of the nuclear membrane (GVB [GV breakdown]) and progress to $\mathrm{MI}$ in 4-6 h. Oocytes extrude the first polar body $(\mathrm{PB})$ at $9-10 \mathrm{~h}$ and arrest at $\mathrm{MII}$ between $10 \mathrm{~h}$ and $12 \mathrm{~h}$ after $\mathrm{LH} / \mathrm{hCG}$ stimulation. (Adapted with permission from Development [from Oh et al. 2000], http:// dev.biologists.org.) (B) Comparison of transcripts associated with polysomes in MI or MII with those recovered from GV oocytes. Transcripts showing less than twofold changes between (constitutively translated, class I) are in yellow, those significantly decreased more than twofold (class II, repressed; $P<0.05$ ) are in blue, and transcripts significantly increased more than twofold (class III, activated) are in red. FDR $<5 \%$ and $P<0.05$ were used in this analysis. $(C)$ Pie chart depicting the relative distribution of transcripts in the three classes using the GV and MII data. The number of the transcripts included in each class is reported. (see the Materials and Methods for inclusion criteria). (D) Stability of class II and class III transcripts during oocyte maturation. The two major classes of regulated transcripts (class $\Pi$ I/repressed and class III/activated) were compared with available databases of total oocyte transcripts (Su et al. 2007). The criteria for classification of stable and unstable transcripts are reported in the Materials and Methods.

mRNAs (Fig. 1D), suggesting that mRNA degradation is a limiting factor for translation of these transcripts. Interestingly, some transcripts decrease early in MI, whereas others decline only later in MII (Supplemental Fig. S5). These divergent time courses indicate that release from the polysomes and/or degradation is transcript-specific and finely regulated. Moreover, $\sim 400$ transcripts remain stable as total transcripts throughout maturation but decrease in the polysome-bound fraction (Fig. 1D). The presence of this distinct subclass was further confirmed by comparing the polysomal and subpolysomal/RNP fractions. The decrease in these transcripts in the polysome mirrored an increase in the subpolysomal/ RNP fractions (Supplemental Fig. S3), confirming that these transcripts are translocated and not degraded. This latter finding implies a second mechanism of translational repression, independent of mRNA degradation.

On average, class III transcripts are stable during oocyte maturation (Fig. 1D). These transcripts recruited to the polysomes code for well-established regulators of the cell cycle such as Ccnb1 and Mos, but also for other cell cycle components (55 transcripts are reported in Supplemental Table S1). These include components of the anaphase-promoting complex (APC) or cyclosome (Apc1, Apc10, Cullin 4b, Cdc20, and Cdc27), components of the spindle assembly checkpoint (Mad2, Bub1b, and Sog12), and components required for chromosome attachment and segregation (Spag1, Spag2, and Espl1). A systematic comparison of the behavior of mRNAs coding for the 12 core components of APC, as well as the associated regulatory proteins, predicts that the composition of the complex is finely regulated at the level of translation (Supplemental Fig. S6). Whereas translation of the regulatory Fzr1/Cdh1 decreases by $70 \%$, Cdc 20 mRNA recovered in the polysomes increases 10-fold, in agreement with the $\mathrm{APC}^{\mathrm{Cdh} 1}$-to-APC ${ }^{\mathrm{Cdc} 20}$ switch that occurs during meiosis (Reis et al. 2007). Cohesin Spag1 and Spag2 mRNA translation is also increased $>10$-fold in $\mathrm{MII}$ oocytes, consistent with a role of these proteins in embryonic divisions. Translational regulation is not limited to proteins involved in the cell cycle, as mRNAs coding for transcription regulators and chromatin remodelers are enriched in class III transcripts $\left(P=4.7^{-9}\right.$ and $\left.3.7^{-5}\right)$ (Supplemental Table S1). Since transcription is silent in maturing oocytes, these nuclear proteins are likely important in the activation of zygotic expression later in development.

\section{Analysis of the 3'UTR of regulated transcripts}

To gain insight into the mechanisms underlying the above patterns of translation, we scanned available 3'UTRs of the three classes of transcripts for enriched motifs using an algorithm we developed, as well as a computational approach used previously to identify conserved sequences in coregulated genes (Grskovic et al. 2007). Several motifs enriched at least fivefold in transcripts recruited to the polysomes were identified using the two unbiased methods (Fig. 2A). The most abundant 


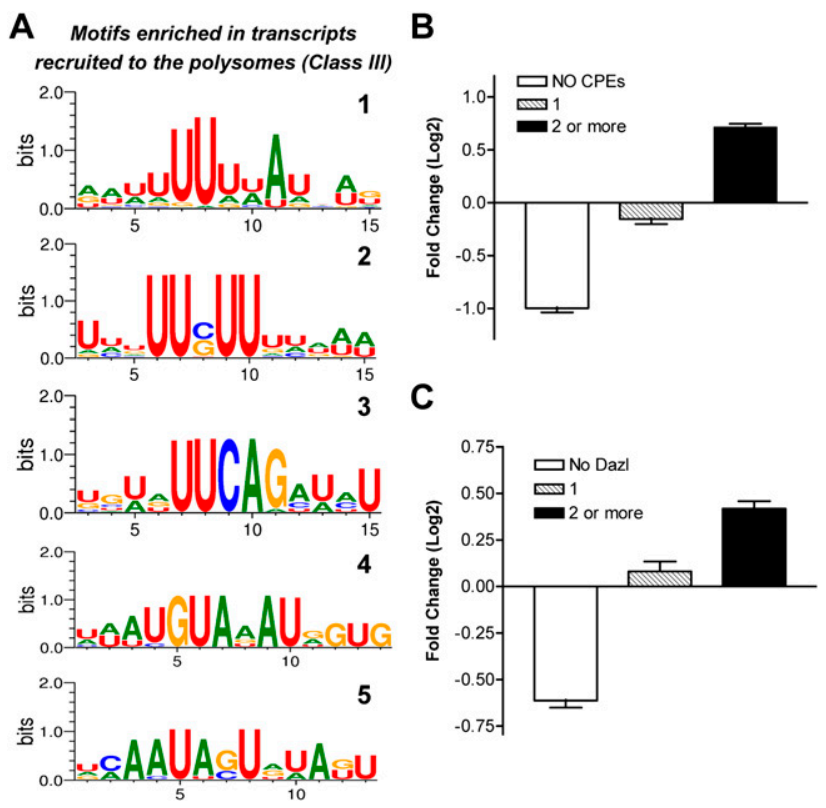

Figure 2. Motifs enriched in the 3'UTR of class III transcripts differentially regulated during oocyte maturation. $(A)$ Sequence logo representation of motifs enriched in class III transcripts. These representative motifs were identified using an unbiased search for sequences enriched in class III transcripts. See the Materials and Methods for details. (B) Relationship between number of CPEs and transcript movement to the polysome fraction. The 3'UTRs of 4645 transcripts were scanned for canonical CPEs (UUUUAU or UUUUAAU) and then subdivided into classes according to the number of putative CPEs detected. The data were compared with a pool of 1954 transcripts without a recognizable CPE and plotted as the $\log _{2}$ of MII/GV fold changes in abundance in the polysome fraction. $(C)$ Relationship between the presence of DAZL elements and transcript recruitment to the polysomes during oocyte maturation. Transcripts were scanned for putative DAZL elements, and transcripts with no DAZL elements (2402 transcripts) or one or two or more Dazl were plotted against the $\log _{2}$-fold GV/MII change in transcript levels in the polysome fraction.

motif enriched in the activated transcripts (Fig. 2A, motif 1) closely matches the $\mathrm{CPE}\left(\mathrm{U}_{4-5} \mathrm{~A}_{1-2} \mathrm{U}\right)$, the canonical target for CPEB1. Together with motifs not related to any known RBPs (Fig. 2A, motif 3), additional elements enriched in transcripts recruited to the polysomes were similar to the consensus binding sites for Puf proteins (Fig. 2A, motif 4) and Musashi (Fig. 2A, motif 5). Finally, some clusters (Fig. 2A, motif 2; Supplemental Table S2) fit the $\mathrm{U}_{2-10}(\mathrm{C} / \mathrm{G}) \mathrm{U}_{2-10}$ consensus sequences for the DAZ family of proteins (Daz, Dazl, and Boule) (Venables et al. 2001; Maegawa et al. 2002; Reynolds et al. 2005).

The above unbiased search for enrichment of elements in the polysome-associated transcripts was refined by scanning 4645 unique 3'UTR sequences from the three classes for the presence of canonical CPE (UUUUA[U/A]U) (Pique et al. 2008) or the previously described loose DAZL consensus sequences (UUU[C/G]UUU) (Venables et al. 2001; Maegawa et al. 2002; Reynolds et al. 2005). Of the 1512 transcripts that do not contain discernible CPE or DAZL-binding elements, $\sim 70 \%$ are decreased in the polysome fraction during oocyte maturation (Supplemental Fig. S9). This underrepresentation in the downregulated transcripts strongly suggests that the absence of CPE or DAZL-binding element is associated with either release from the polysomes or destabilization of a transcript. Among the remaining sequences, one or more of the two putative RBP consensus sites (see Supplemental Table S3) was identified. The presence of two or more CPE or DAZL-binding elements was associated with transcripts that, on average, increased in the polysome fraction during maturation to MII (Fig. 2B,C; Supplemental Fig. S9). The simultaneous presence of both CPEs and DAZL-binding elements was observed in 1799 transcripts; on average, these were increased in the polysome fraction during maturation (Supplemental Fig. S9). To explain the temporally distinct waves of translation during Xenopus oocyte maturation, a combinatorial code involving the CPE has been proposed: Two closely positioned CPEs are required for repression, whereas 25 nucleotides (nt) is the distance of a single CPE from the hexamer optimal for polyadenylation and translational activation (Pique et al. 2008). To test whether the 3'UTRs of transcripts recruited to the polysomes conform to this rule, we investigated the CPE position in different classes of transcripts. A tendency toward an enrichment of CPEs in the proximity of the hexamer was observed in the oocyte transcripts (Supplemental Fig. S7); however, no statistically significant correlation between polysome recruitment of these endogenous transcripts and distance from the hexamer could be established. Nevertheless, multiple putative CPEs are more frequently associated with translated transcripts, whereas a single CPE is neutral (Fig. 2B; Supplemental Figs. S8, S9), confirming a link between CPEs and translation of a subset of MII transcripts in mouse oocytes.

The above data strongly suggest that combined or hierarchical functions of several RNA regulators orchestrate maternal mRNA translation in MII. This possibility was explored by comparing the translation of proteins interacting with the above elements. Interestingly, the polysome data showed opposing patterns of translation for Cpeb1 mRNA, down-regulated during maturation, compared with other RBP transcripts, such as Dazl, Pum2, Cpeb3, and Cpeb4, which are up-regulated (Supplemental Fig. S10). A preliminary screening for the function of Cpeb1, Cpeb3, Cpeb4, Dazl, and Pumilio2 was performed by injection of specific antisense morpholino olignucleotides (MOs) in GV oocytes, and progress through maturation was used as readout. Whereas Cpeb3 and Cpeb4 MOs had no significant effect, Cpeb1, Dazl, and Pumilio2 MOs significantly decreased $(\sim 50 \%)$ progression through meiosis (Supplemental Fig. S11). On the basis of this initial screening, the relationship between Cpeb1 and Dazl was explored further.

\section{Regulation of DAZL synthesis during oocyte maturation}

qPCR of independent samples confirmed the recruitment of Dazl mRNA to the polysome, whereas Dazl overall 
transcript levels are unchanged (Fig. 3A). This translocation was associated with a proportionate decrease in Dazl mRNA levels in the subpolysomal/RNP fraction (Fig. 3A). Western blot analysis of oocyte extracts showed a fivefold increase in DAZL protein levels at progressive stages of development (up to MII and zygote), followed by an abrupt decrease in the two-cell embryo (Fig. 3B, C), confirming that recruitment to the polysomes is tightly coupled to translation of this protein. In agreement with the pattern of Cpeb1 mRNA association with the polysomes (Supplemental Fig. S10), CPEB1 protein is present in GV oocytes, remains stable up to $4 \mathrm{~h}$, shifts in electrophoretic mobility between 4 and $6 \mathrm{~h}$, and becomes undetectable thereafter (Fig. 3C; Supplemental Fig. $\mathrm{S} 4 \mathrm{~A}, \mathrm{E})$. This latter finding in mouse oocytes is reminiscent of Xenopus Cpeb1, which is phosphorylated by $C d c 2$ and then targeted to the proteasome for degradation (Mendez et al. 2002; Setoyama et al. 2007).

The 3'UTR of Dazl mRNA contains putative CPEs. Indeed, Dazl mRNA is immunoprecipitated by CPEB1specific antibody (Fig. 4A), indicating a physical interaction between CPEB1 and Dazl transcript in mouse

A

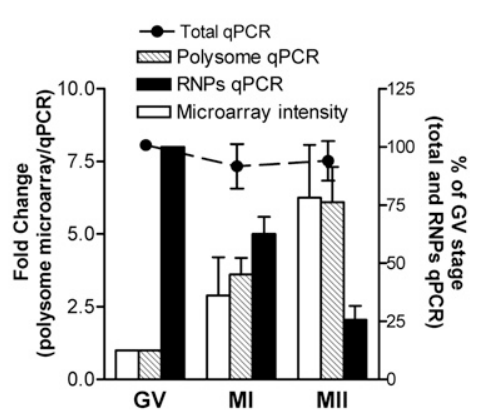

B

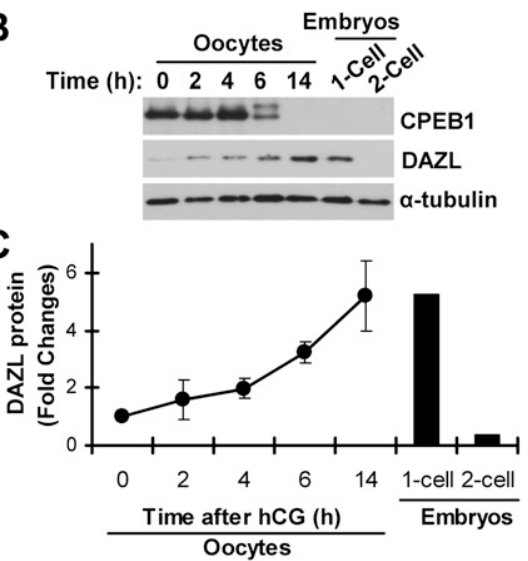

Figure 3. Dazl mRNA translation and protein accumulation during maturation. (A) qPCR analysis of Dazl mRNA in polysome and subpolysome/RNP fractions and whole-cell lysates during oocyte maturation. Each point is the mean \pm SEM of three to five biologically different samples. The microarray data are included for comparison. (B) DAZL protein accumulation at the oocyte-to-zygote transition. A representative experiment of the four experiments performed is reported. Accumulation of CPEB1 and $\alpha$-tubulin was used as a control. $(C)$ Quantification of the intensity of the DAZL immunoreactive band from different experiments (mean \pm SEM; $N=4$ ). oocytes. Furthermore, the poly(A) length of Dazl mRNA increases from 50 to $150 \mathrm{nt}$ during the GV-MI transition, and then the mRNA is partially deadenylated in MII (Fig. 4B). When CPEB1 is down-regulated by MO injection, Dazl polyadenylation is disrupted in MI stage oocytes (Fig. 4C) and the accumulation of DAZL protein in MII oocytes is significantly reduced (Fig. 4D). In addition to CPEs, the Dazl 3'UTR contains at least 12 sequences that fit the Dazl consensus; because mutation of all of these motifs would significantly alter the 3'UTR sequence, the functionality of these putative DAZL-binding elements was assessed by two alternative approaches. DAZL knockdown by MOs markedly reduces the late translation of a reporter fused with the Dazl 3'UTR (Fig. 4E). More importantly, DAZL binds specifically to its own mRNA, as assessed by oocyte extract immunoprecipitation with DAZL antibodies (Fig. 5A). These findings strongly suggest that, during the GV-MI transition, Cpeb1 promotes the initial Dazl polyadenylation and translation, and newly synthesized DAZL then increases translation of its own mRNA, establishing a self-reinforcing, positive feedback loop.

Work done in C. elegans, Xenopus, and male mice indicates that Dazl functions as a translational activator, and several putative targets have been identified (Reynolds et al. 2005, 2007). In agreement with these findings, immunoprecipitation of MI oocyte extracts with DAZL antibodies lead to $\sim 10$-fold enrichment of several transcripts, including those coding for Dazl itself, for the cell cycle regulators $B u b 1 b, C d c 20$, and $T p \times 2$; chromatin remodelers Arid1A and Smarca5; and Tex19.1 (Fig. 5A). Tex19.1 has been reported as a potential target of DAZL during spermatogenesis (Reynolds et al. 2005). Because the abundance of this mRNA in the polysome fraction increases markedly during oocyte maturation (Fig. 5B,C), and only four putative DAZL-binding elements are present in the 3'UTR, this target was used to test the involvement of the DAZL protein in translational activation. Consistent with the polysome recruitment, the luciferase activity is increased when the reporter under the control of the Tex19.1 3'UTR is expressed in oocytes (Fig. 5E). More importantly, mutation of the putative Dazl elements in this 3'UTR or down-regulation by Dazl MOs prevents the increase in luciferase activity (Fig. 5E,F). Taken together, these findings provide experimental evidence that DAZL binds and regulates the translation of target mRNAs in oocytes during late maturation.

\section{$D A Z L$ protein accumulation is essential for oocyte maturation and early embryo development}

As mice deficient in Dazl are sterile and devoid of germ cells (Ruggiu et al. 1997; Lin and Page 2005), a role for this protein in the final stages of oocyte maturation has not been investigated. We probed the Dazl function at the oocyte-to-zygote transition by suppressing Dazl mRNA translation with MOs. Injection of Dazl MOs in GV oocytes completely prevented the increase in DAZL protein in MII (Fig. 6A). This disruption causes a delay in meiotic resumption (Fig. 6B); reduces the number of 
Chen et al.

A
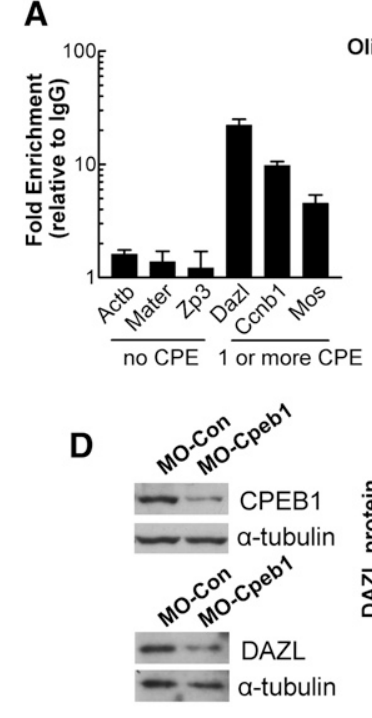

B

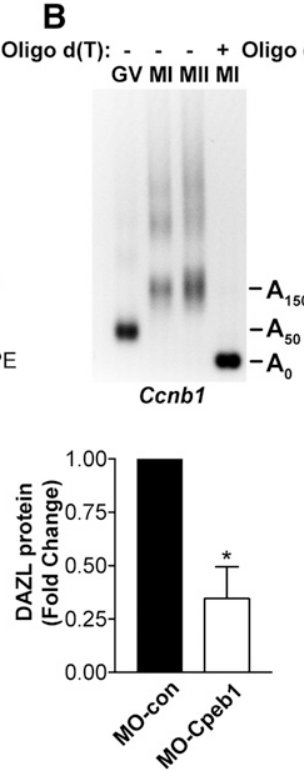

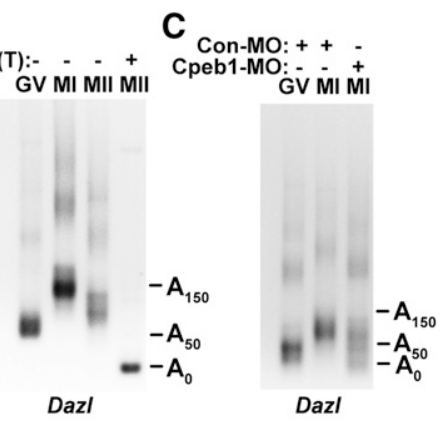

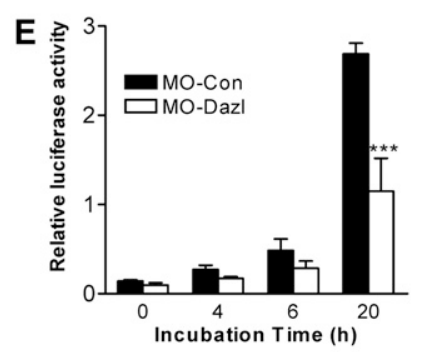

Figure 4. Regulation of the Dazl mRNA translation during oocyte maturation. (A) CPEB1 protein coimmunoprecipitates with Dazl transcripts. GV stage oocytes were lysed and extracts were immunoprecipitated with nonimmune IgG or CPEB1 antibodies. mRNAs recovered in the immunoprecipitation pellets were quantitated by qPCR. The data are corrected for the IgG background and reported as the mean \pm SEM of three separate experiments. (B) Polyadenylation of Ccnb1 and Dazl during oocyte maturation in vivo. After stimulation with hCG, GV, MI, and MII stage oocytes were isolated and used for PAT assays. A representative experiment of the three experiments performed is reported. $(C)$ Polyadenylation of Dazl mRNA in MI is disrupted in Cpeb1 MO-injected oocytes. After injection, oocytes were preincubated in $2 \mu \mathrm{M}$ Milrinone overnight, then cultured in inhibitor-free medium to induce maturation. Samples were collected at indicated stages and used for PAT assays. (D) DAZL protein accumulation depends on CPEB1. GV stage oocytes and oocytes with polar bodies were collected to assess CPEB1 and DAZL protein levels, respectively. The bar graph on the right reports the densitometric quantification of the DAZL signal (mean \pm SEM, $N=3$ ). (E) Dazl mRNA autoregulation. RNA from a construct with renilla luciferase (RL) coding region fused to the 3'UTR of Dazl was coinjected in GV oocytes with either control MOs or Dazl MOs. Oocytes were collected at different times of maturation and extracts were assayed for luciferase activity $(\mathrm{mean} \pm \mathrm{SEM} ;=4)$.

oocytes that complete meiosis I, measured as polar body extrusion (Fig. 6C); and disrupts the transition to MII (Fig. 6D). Although a polar body is extruded in $\sim 35 \%$ of the oocytes injected with Dazl MOs, the MII spindle is absent or defective in $90 \%$ of knockdown oocytes, and, in most cases, microtubules remain dispersed throughout the oocyte cytoplasm (Fig. 7A,B). Chromosome condensation and congression are also disrupted (Fig. 7A). A defective
MII was confirmed functionally by the finding that oocytes injected with Dazl MOs cannot be fertilized (Fig. 6D). The MI-arrested phenotype was rescued by coinjection of either human recombinant DAZL protein (Figs. 6C, 7C; Supplemental Fig. S12) or human Dazl mRNA (Supplemental Fig. S12). The sequence around the ATG of human Dazl is substantially different from that of the mouse, and therefore should not be the target for the
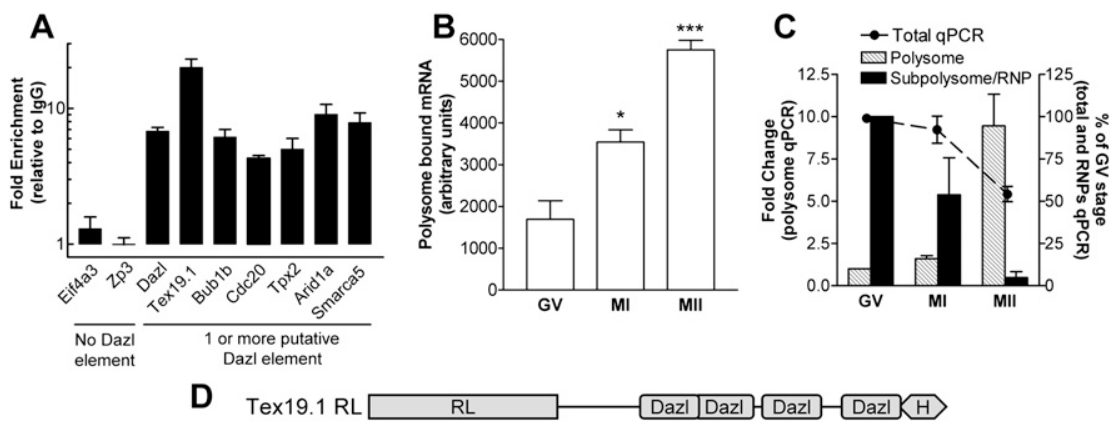

$$
\begin{aligned}
& \text { D Tex19.1 RL } \\
& \text { Tex19.1 Del-RL }
\end{aligned}
$$
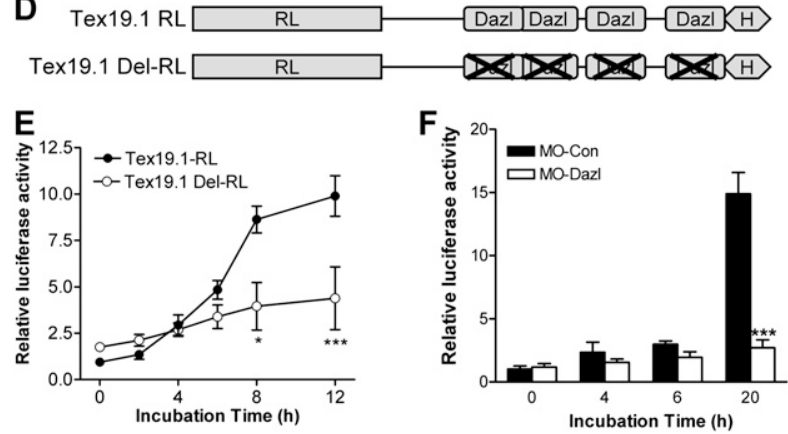

Figure 5. DAZL is involved in the translation of Tex19.1. (A) Transcripts enriched in DAZL immunoprecipitates of oocyte extracts. After stimulation with hCG, MI stage oocytes were harvested as described. Oocyte lysates were immunoprecipitated with DAZLspecific antibody and selected transcripts were detected by qPCR. $(B, C)$ Tex19.1 is recruited to the polysomes during oocyte maturation. Microarray analysis $(B)$ and qPCR $(C)$ of Tex19.1 mRNA in the polysome, subpolysome/RNP fractions, and total transcripts are shown. $(D)$ Diagram of the luciferase constructs injected in oocytes. (E) Mutation of DAZL-binding elements impaired the expression of reporter during oocyte maturation. After overnight incubation, oocytes were cultured in inhibitor-free medium to allow maturation. Samples were collected at indicated culture times for assays of luciferase activity. (F) Decreased expression of Tex19.1-RL in DAZL knockdown oocytes. Luciferase activity assay was performed in extracts from oocytes coinjected with Tex19.1-RL and Dazl MOs or control MOs. 
A
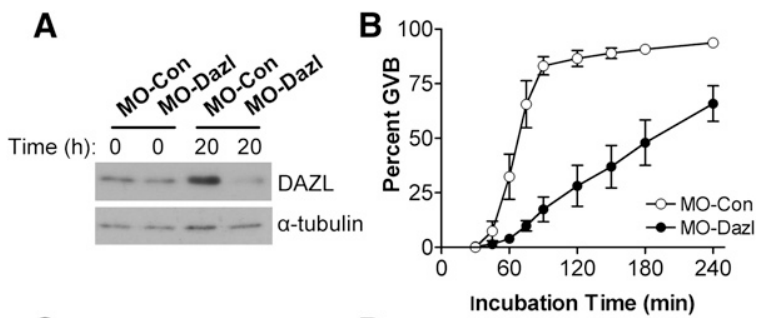

C

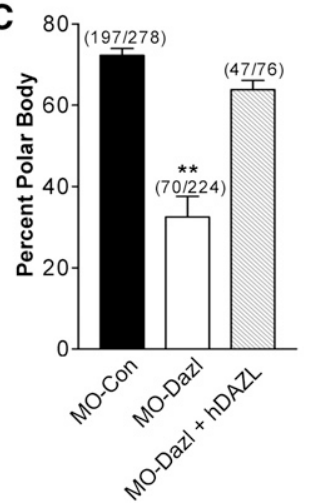

D

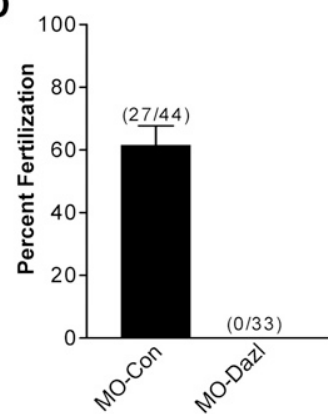

Figure 6. Dazl is required for oocyte maturation and early embryo development. (A) Morpholino down-regulation of DAZL protein. Oocytes injected with control or Dazl MOs were preincubated overnight with $2 \mu \mathrm{M}$ Milrinone and then cultured in inhibitor-free medium for maturation. Oocytes were collected at indicated times and used for Western analysis to detect DAZL protein levels. A representative experiment of the five experiments performed is reported. $(B)$ Oocytes injected with Dazl MOs show a slow GVB time course. $(C)$ Decreased polar body extrusion in Dazl-deficient oocytes. A group of oocytes was also injected with human DAZL protein. The number above the bar indicates the total number of oocytes injected in each group and the number of oocytes extruding a polar body. (D) Impaired fertilization in Dazl-deficient oocytes. Cumulus cell-enclosed oocytes were injected with control or Dazl MOs. After $15-16 \mathrm{~h}$ of incubation, they were used for in vitro fertilization. The fertilization rate was assessed by scoring the number of two-cell embryos after an additional overnight culture.

MOs (Supplemental Fig. S12). A partial rescue also was observed for the MII spindle phenotype (Fig. 7B,C; Supplemental Fig. S13A). When early zygotes are injected with Dazl MOs, an almost complete block at the two-cell stage was observed (Supplemental Fig. S15). This phenotype was rescued by coinjection of human Dazl mRNA. Consistent with the absence of DAZL at the two-cell stage, no effects were observed when injection in the embryo was delayed to the late zygote stage /data not shown). Surprisingly, immunolocalization of the endogenous DAZL shows, together with a diffuse distribution throughout the oocyte cytoplasm, a symmetrical enrichment at the poles of both MI and MII metaphase spindles (Fig. 7C; Supplemental Fig. S13A)-localization that overlaps with that of pericentrin (Supplemental Fig. S13B). This localization is lost after Dazl MO injection (Fig. 7A), as is the diffuse DAZL staining in the cytoplasm of the oocyte.

TPX2 (targeting protein for the Xenopus kinesin xklp2) is required for spindle assembly during oocyte maturation, and Tpx2 down-regulation causes a phenotype rem-

iniscent of Dazl knockdown (Brunet et al. 2008). Tpx2 3'UTR contains multiple DAZL-binding elements, and Tpx2 is enriched in DAZL immunoprecipitation of oocyte extracts (see Fig. 5A). Whereas Tpx2 translation is increased in MI/MII (Fig. 7D), MO knockdown of Dazl is associated with decreased accumulation of this microtubule regulator (Fig. 7E), and the expression of luciferase reporter fused to the Tpx2 3'UTR was significantly decreased in these oocytes (Fig. 7F). These data strongly suggest that DAZL regulates Tpx2 mRNA translation during maturation, and that TPX2 accumulation is disrupted in Dazl knockdown oocytes.

\section{Discussion}

The above studies provide a genome-wide representation of transcripts associated with polysomes at different steps of oocyte maturation, revealing a widespread involvement of regulated translation in cell cycle control and other biological processes. Analysis of these data demonstrates the presence of a novel regulatory circuit involving two RNA modulators. We showed that CPEB1 activates the translation of Dazl mRNA during MI, which in turn directs its own translation as well as that of a subset of mRNAs during later stages of oocyte development. These DAZL-dependent translations are necessary for spindle assembly, the MI-MII transition, and early embryo development. Thus, temporally distinct waves of translation at different stages of oocyte maturation are generated via positive, reinforcing loops of these interacting RBPs.

It is well established that accumulation of some key components of the cell cycle-such as Ccnb1, Mos, and Wee2-are regulated at the level of translation in frog oocytes (Richter 1991, 2007). Here we confirm that translational regulation of these transcripts occurs in mouse oocytes. More importantly, we found that translation of a large number of other cell cycle components is also positively or negatively regulated in phase with the meiotic transitions. This observation adds a new dimension to the control of cell division, which is widely believed to be mediated by post-translational modifications. Finely regulated translation and protein synthesis coexist and cooperate with phosphorylation and APCmediated protein degradation to control each phase of the two meiotic divisions.

Our data demonstrate that the interplay between two RNA regulators, CPEB1 and DAZL, is an essential component of the translational program in mouse oocytes. CPEB1 binds to Dazl mRNA and is required for its polyadenylation and the initial accumulation of the DAZL protein in MI. Additional delayed translation of Dazl mRNA in MI/MII requires the accumulation of DAZL itself, establishing a self-reinforcing, positive feedback loop. A similar autoregulation of Dazl mRNA has been reported in Danio rerio (Takeda et al. 2009). On the basis of these findings, we propose the following model of translational regulation in mouse oocyte meiosis (Supplemental Fig. S16). During the prophase-to-MI transition, CPEBl activates the polyadenylation of Dazl mRNA, 

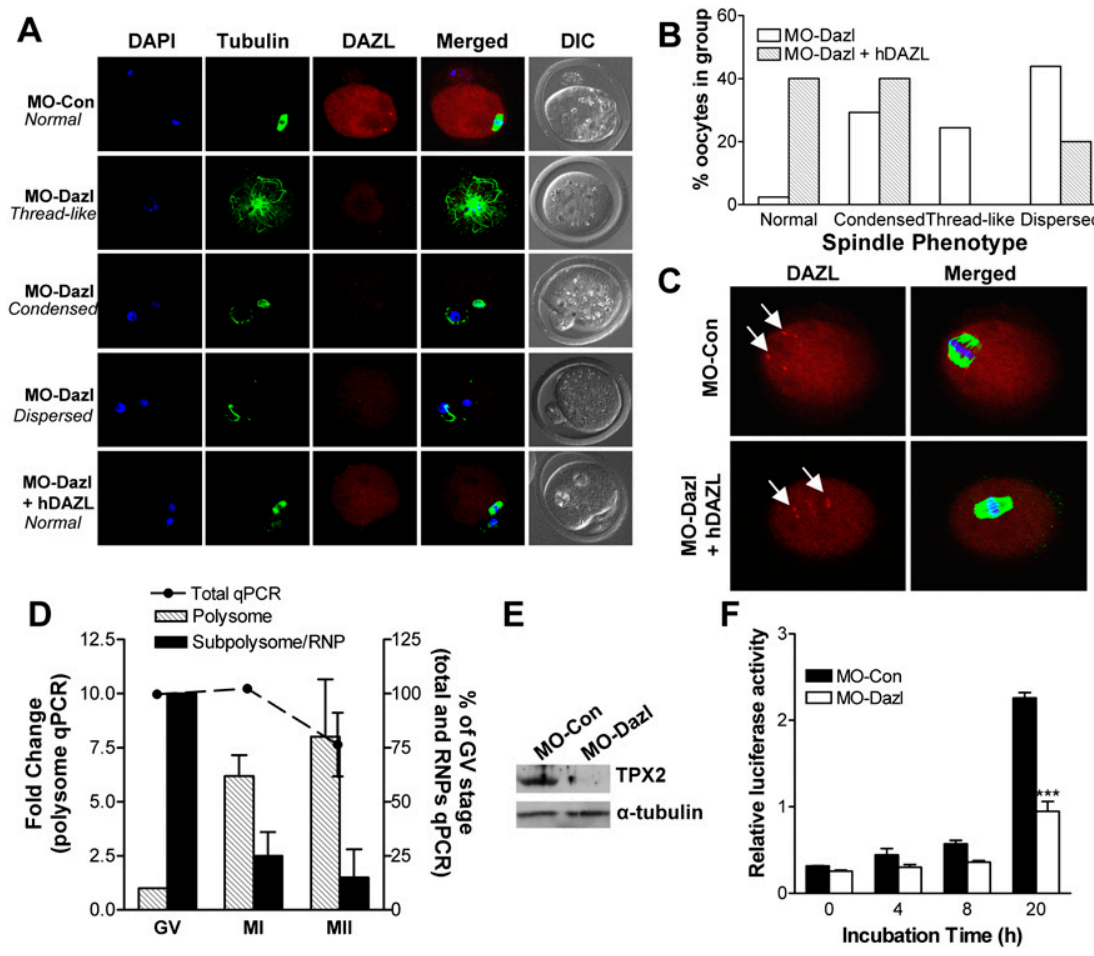

Figure 7. Spindle localization and defective spindle formation in Dazl-deficient oocytes. (A) Representative patterns of DNA, tubulin, and DAZL localization in oocytes injected with control MOs, Dazl MOs, or Dazl MOs plus hDAZL protein. (B) Quantification of spindle phenotypes in oocytes injected with Dazl MOs in the presence or absence of hDAZL protein. $(C)$ Details of spindle localization of DAZL. (D) qPCR of Tpx2 mRNA in the polysome and subpolysome/RNP fractions and total transcripts are shown. (E) TPX2 expression during meiosis is dependent on Dazl. Oocytes were injected with control MOs or Dazl MOs and incubated in meiotic arresting medium for $20 \mathrm{~h}$. Oocytes were then cultured in medium that allows maturation overnight and collected for Western blot analysis using antibodies against TPX2 and $\alpha$-tubulin. A representative experiment of the three experiments performed is reported. $(F)$ Dazl is required for translation of a RL reporter fused to the 3'UTR of Tpx2. Luciferase activity was measured in extracts from oocytes coinjected with Tpx2-RL and Dazl MOs or control MOs. The data are mean \pm SEM of three independent experiments. thereby promoting its translation. This initial increase becomes self-sustained and extended in time through a positive feedback regulation of Dazl mRNA. The accumulating DAZL protein in turn activates the translation of a subset of class III transcripts such as Tex19.1, $T p \times 2$, and other regulators of the cell cycle. This arrangement of two RBPs acting sequentially allows late translations in MII, in spite of the fact that CPEB1 is degraded earlier during anaphase of the first meiotic division. This model provides a mechanistic explanation of how waves of translations are produced synchronously with the cell cycle. Interestingly, translation of Cpeb3 and Cpeb4 mRNAs were up-regulated in mouse oocytes during maturation. Even though MO injection had no detectable effect on MI progression, it cannot be excluded that these CPEB1 orthologs play a role in late CPE-mediated polyadenylations in MII. In the same vein, a recent study has shown that CPEB4 replaced CPEB1 to sustain cytoplasmic polyadenylation in frog oocytes (Igea and Mendez 2010). Thus, it is possible that CPEB4- and DAZL-mediated regulations cooperate in the late translation of mouse oocyte transcripts containing both consensus sites.

Down-regulation of DAZL disrupts the assembly of meiotic spindles in MI and MII, thus compromising meiotic progression. Immunoprecipitation of DAZL protein from oocyte extracts demonstrates an interaction with components of APC $(C d c 20)$ and spindle assembly checkpoint $(B u b 1 b)$, as well as spindle components such as Tpx2, suggesting that Dazl controls translation of critical components of spindle function. TPX2 targets spindle microtubules and activates Aurora A, an essential regulator of centrosome and spindle pole assembly (Bayliss et al. 2003; Eyers and Maller 2004). In addition, TPX2 induces a Ran-GTP-dependent microtubule nucleation in the vicinity of chromosomes and participates in spindle pole organization (Gruss et al. 2001). Our studies provide evidence that $T p \times 2$ is a bona fide target for DAZLmediated translation in oocytes, as its accumulation requires DAZL. Tpx2 MO down-regulation causes phenotypic changes very similar to those we observed with Dazl knockdown (Brunet et al. 2008), including disruption of spindle assembly and failure of chromosome condensation. Therefore, defective activation of Aurora $A$ and spindle function caused by inadequate levels of TPX2 protein is likely to contribute to the defects we observed after Dazl knockdown. A loss of compartmentalized Dazl-dependent translation may be an additional cause of the phenotypes we observed. Indeed, it has been reported that localized translation occurs in the proximity of the spindle microtubules (Blower et al. 2007). In Xenopus oocytes, spindle-localized CPEB regulates translation and controls chromosome segregation (Eliscovich et al. 2008). Together with diffuse localization in the oocyte cytoplasm, DAZL is enriched at the spindle poles with a localization that overlaps with that of pericentrin. Thus, it is possible that DAZL targeting to these regions provides a means to localize mRNAs and perhaps synthesize spindle proteins, consistent with the concept that spatial regulation of gene expression is required for oocyte development (King et al. 2005).

We show that increased translation of at least three mRNAs (Tex19.1, Tpx2, and Dazl itself) is dependent on DAZL protein accumulation, supporting the hypothesis that this RBP functions as a translational activator. However, the exact molecular mechanism by which DAZL promotes translation remains to be determined. 
It has been proposed that DAZL is required for translation initiation (Collier et al. 2005) by promoting recruitment of the poly(A)-binding proteins PABP1 and ePABP in the absence of poly(A) elongation. These, in turn, promote translation by interacting with the CAP complex. In frog oocytes, Dazl is not as effective in stimulating translation of transcripts that are already polyadenylated (Collier et al. 2005), suggesting that DAZL action is decreased when PABPs are already recruited on the mRNA. Consistent with the above model, we found that maximum DAZL protein accumulation and translation of the Dazl reporter occur at a time when Dazl mRNA is partially deadenylated. In contrast to the above findings, it has been reported that, in zebrafish, Dazl promotes lengthening of the poly(A) tail by interfering with microRNA (miRNA) repression (Takeda et al. 2009). It is unlikely that this regulatory mechanism functions in mature mouse oocytes in view of the recent reports that miRNA function is inactivated at this stage of oocyte development (Ma et al. 2010; Suh et al. 2010). At variance with our finding that both $3^{\prime} \mathrm{UTR}$ mutagenesis and MO downregulation of DAZL decrease Tex19.1 translation, a study suggested that Dazl represses Tex19.1 translation when introduced in zebrafish oocytes (Zeng et al. 2009). Although removal of DAZL elements from the $3^{\prime}$ UTR of Tex19.1 impairs accumulation of the reporter in MII oocytes, it also causes a small increase in reporter translation in GV, suggesting that this element may mediate translational repression at this stage. DAZL is known to interact with several other proteins, including Pumilio 2, which may function as a translation repressor (Brook et al. 2009). Thus, it is possible that DAZL interaction with other RBPs defines whether translation of a target mRNA is repressed or activated.

A Dazl requirement for completion of female meiosis is reminiscent of the function of the Drosophila ortholog Boule during male meiosis, where it is necessary for the G2/M transition and expression of the cell cycle component twine/Cdc25 (Maines and Wasserman 1999). This homology lends support to the hypothesis that cell cycle regulation is a shared function for this family of proteins, a view that may provide a novel mechanistic insight into the role of Dazl during PGC proliferation and early gonadogenesis. Furthermore, the possibility that Cpeb1 is epistatic to Dazl also during PGC entry into meiosis is consistent with several observations. Cpeb1 knockout causes a disruption of prophase and failure to accumulate components of the synaptonemal complex such as SYCP3 (Tay and Richter 2001), which is a direct target for Dazl (Reynolds et al. 2007; Lin et al. 2008). Although a direct interaction between DAZL and CPEB1 could not be detected, a sequential control of Sycp3 translation by CPEB1 and DAZL has been proposed during spermatogenesis (Reynolds et al. 2007). Given our findings in the oocyte, a functional interaction between DAZL and CPEB1 in the regulation of many mRNA targets during fetal gonadal development warrants further investigation. Taken together, all of these findings strongly suggest that DAZL plays a critical function at different steps of meiosis and gamete development.
Finally, the demonstration that DAZL is a component of an RBP network that controls maternal mRNA translation provides a mechanistic insight into the acquisition of oocyte developmental competence. The coordinated recruitment or release from translating polysomes of subsets of maternal mRNAs is likely essential for assembling the maternal machinery required for the initial stages of embryo development until the zygote genome becomes activated. This is in line with the concept that embryogenesis begins during oogenesis (Wolpert et al. 2002). Together with the analysis of polysome mRNA reported at the MII/zygote transition (Potireddy et al. 2006) the genome-wide analysis of translation that we performed should provide a blueprint of the events that, in the embryo, are critical for fertilization, nuclear reprogramming to totipotency, and activation of the embryonic genome.

\section{Materials and methods}

\section{Polysome isolation and microarray analysis}

C57BL/6 female mice (22-24 d old) were used in all experiments. Forty-eight hours after PMSG injection, mice were stimulated with hCG for $0 \mathrm{~h}, 4 \mathrm{~h}$, or $14 \mathrm{~h}$, and GV, MI, and MII stage oocytes were collected. Approximately 500-600 oocytes were lysed with $1 \times$ PLB $(30 \mathrm{mM}$ Tris- $\mathrm{HCl}$ at $\mathrm{pH} 7.5,100 \mathrm{mM} \mathrm{NaCl}, 10 \mathrm{mM}$ $\mathrm{MgCl}_{2}, 1 \%$ Triton, $1 \mathrm{mM}$ DTT, $0.25 \mathrm{mM} \mathrm{Na}_{3} \mathrm{VO}_{4}, 20 \mathrm{mM}$ $\beta$-glycerophosphate, $30 \mathrm{U} / \mathrm{mL}$ RNase inhibitor [USB Chemical], $10 \mu \mathrm{g} / \mathrm{mL}$ cycloheximide, plus protease inhibitor cocktail [Roche]). Oocyte lysates were centrifuged at $12,000 \mathrm{~g}$ for $10 \mathrm{~min}$ at $4^{\circ} \mathrm{C}$. Supernatants were loaded on a $10-\mathrm{mL} 15 \%-50 \%$ sucrose gradient and centrifuged at $100,000 \mathrm{~g}$ for $110 \mathrm{~min}$ at $4^{\circ} \mathrm{C}$. RNAs from the polysome or RNP fractions were precipitated with ethanol overnight and purified with RNeasy Plus Micro kit (Qiagen). RNAs in the RNP fractions were reverse-transcribed with SuperScript III (Invitrogen). Polysome-bound RNAs were reverse-transcribed and linearly amplified with WT-Ovation FFPE RNA Amplification System version 2 (NuGEN). Five micrograms of cDNA was fragmented and hybridized with Affymetrix Mouse Genome 430.2 array chips. Microarray data for transcripts isolated from the whole oocytes were obtained from data deposited in Gene Expression Omnibus (GEO) (Su et al. 2007). DNA-Chip Analyzer (dChip) was used for normalization and to quantify microarray signals with default analysis parameters. Data were then filtered for presence/absence calls, and transcripts with an intensity of $<100$ in all three stages were considered absent from the polysome fraction and were removed before further analysis. Comparison between each sample group was performed using dChip with a fold change of \pm 2 or more, FDR $<5 \%$, and $P<0.05$. Transcripts failing to pass this criteria were considered constitutively translated (polysome-bound transcripts) or stable (total transcripts). All gene identifiers and their corresponding $P$-values and fold changes were uploaded into Genmapp 2.1 (gene map annotator and pathway profiler). Gene ontology, biological processes, and molecular function analyses were performed using protein analysis through evolutionary relationships (PANTHER) or Genmapp.

\section{Oocyte culture and microinjection}

The culture medium used for most experiments was Eagle's minimum essential medium with Earle's salts (MEM, GIBCO), supplemented with $0.23 \mathrm{mM}$ pyruvate, $75 \mu \mathrm{g} / \mathrm{mL}$ penicillin, 10 
$\mu \mathrm{g} / \mathrm{mL}$ streptomycin sulfate, and $3 \mathrm{mg} / \mathrm{mL}$ BSA, and buffered with $26 \mathrm{mM}$ sodium bicarbonate. For oocyte isolation and microinjection, the medium was supplemented with $5 \mu \mathrm{M}$ cilostamide and buffered with $25 \mathrm{mM}$ HEPES,(pH 7.2), with sodium bicarbonate reduced to $6 \mathrm{mM}$. Denuded oocytes were injected with 5- to 10-pL samples per oocyte. After injection, oocytes were washed and cultured in MEM/BSA plus $2 \mu \mathrm{M}$ Milrinone at $37^{\circ} \mathrm{C}$ with $5 \% \mathrm{CO}_{2}$.

\section{Real-time $q P C R$}

Real-time qPCR was performed using Power SYBR PCR master mix with ABI 7900 Real-Time PCR system (Applied Biosystems). All primers were designed in two exons flanking introns to avoid amplification of genomic DNA (Supplemental Table S4). A dissociation curve analysis was performed at the end of the amplification to verify the specificity of the primers. The polysome data were normalized to Eif4a1 and Hsp90ab1 transcript abundance, and the subpolysome/RNP and total transcript data were corrected for oocyte number. REST 2008 software was used to analyze the data.

\section{Motif analysis}

Each transcript from the different groups was subdivided into all possible subsequences of a particular length. For example, for $n=5$, the sequences $1-5,2-6,3-7$, etc., were generated. The outcome of this script was a dictionary with each subsequence associated with a count of the number of times it was found in the experimental and control sets. This was repeated for different lengths of subsequences with $n=5-12$. At the end of this procedure, all subsequences, hereafter referred to as motifs, were filtered to find motifs present more than zero times in control, more than five times in the experimental, and where experiment/control was more than four times (number of times the element is found in experiments/total length of sequence in experiments)/(times found in control/total length of sequence in control). This filtered set of enriched motifs was then submitted to a clustering analysis. The clustering analysis consisted of a simple algorithm whereby all enriched motifs were compared pairwise to each other. If two motifs had two or fewer mismatches, they were placed together in a cluster. For motifs of unequal length, two or fewer mismatches in the smaller of the two motifs was set as the criteria for clustering. This algorithm was used to generate clusters of related motifs. The clusters of related motifs were aligned using ClustalW2 (http://www.ebi. ac.uk/Tools/clustalw2/index.html), and the alignments were submitted to the WebLogo 3 server (http://weblogo.threeplusone. com// using the default DNA/RNA option.

For a targeted search for CPE and DAZL elements, all oocyteexpressed sequences were parsed by identifying the longest 3'UTRs. The resulting 4645 3'UTR sequences included 2500 unchanged, 1148 down-regulated, and 998 up-regulated sequences. These sequences were searched for CPE (UUUUA[U/ $\mathrm{A}] \mathrm{U})$ and Dazl $\left(\mathrm{U}_{2-3}[\mathrm{C} / \mathrm{G}] \mathrm{U}_{2-3}\right)$ consensus sites. This search also identified sequences containing both CPEs and DAZL elements (1799 sequences).

\section{In vitro fertilization}

Cumulus cell-enclosed oocytes were isolated in HEPES-buffered MEM/BSA ( $3 \mathrm{mg} / \mathrm{mL})$ and microinjected with indicated MOs. After injection, oocytes were washed and cultured in MEM $\alpha$ (GIBCO) plus $1 \mathrm{ng} / \mathrm{mL}$ EGF, $1 \mathrm{mg} / \mathrm{mL}$ Fetuin, and $3 \mathrm{mg} / \mathrm{mL}$ BSA at $37^{\circ} \mathrm{C}$ with $5 \% \mathrm{CO}_{2}$. After $16-17 \mathrm{~h}$ of maturation, in vitro fertilization was carried out in MEM/BSA without Fetuin or EGF.
Fertilization was determined by monitoring pronuclear formation and scoring the number of two-cell embryos.

\section{Western blot analysis}

Oocyte lysates were applied to SDS-PAGE (10\% Tris-glycine gels) and transferred to PVDF membranes. Membranes were blocked in $5 \%$ milk (TBS at $\mathrm{pH} 7.4$ ) for $2 \mathrm{~h}$ at room temperature and incubated with primary antibody $(1: 500)$ overnight at $4{ }^{\circ} \mathrm{C}$. After washing in TBS-Tween $20(0.05 \%)$, membranes were incubated with HRP-conjugated secondary antibodies (1:2000; Pierce) for $1 \mathrm{~h}$ at room temperature. Signals were detected using Super Signal Dura (Pierce). Antibodies against CPEB1 and DAZL were from Abcam, TPX2 antibody was from Novus, and CCNB1 (Cyclin B1) antibody was from Cell Signaling. The $\alpha$-tubulin antibody was from Sigma.

\section{Immunofluorescent staining}

Oocytes were fixed in $2 \%$ paraformaldehyde in PBS for $30 \mathrm{~min}$ and permeabilized in $0.1 \%$ Triton X-100 in PBS for 15 min at room temperature. Oocytes were then washed and blocked in PBS containing $0.1 \%$ BSA and $0.01 \%$ Tween- 20 for at least 15 min, and then stained with DAZL antibody (1:100 dilution) and $\alpha$-tubulin antibody (1:500 dilution) or pericentrin antibody (1:100; BD Transduction Laboratory) for $1 \mathrm{~h}$ at room temperature. After washing three times with blocking solution, the oocytes were incubated for $1 \mathrm{~h}$ with Alexa Fluor 488 goat anti-mouse (1:500 dilution; Molecular Probes) and Alexa Fluor 594 goat antirabbit (1:500 dilution; Molecular Probes). Ten micromolar 4,6 diamidino-2-phenylindole dilactate (DAPI) (Molecular Probes) was added to the secondary antibody solution to stain DNA. Oocytes were mounted in CITIFLUOR (Anti-fadent Mounting Medium, AF3, Electron Microscopy Sciences) and imaged on a Leica TCS SP5 confocal microscope.

\section{Immunoprecipitation of mRNA-protein complexes}

Approximately $150 \mathrm{GV}$ or MI oocytes were lysed using $1 \times$ PLB. After centrifugation at $12,000 \mathrm{~g}$ for $10 \mathrm{~min}$, supernatants were precleared with protein G-Separose beads (Invitrogen) for $2 \mathrm{~h}$ at $4^{\circ} \mathrm{C}$. The precleared lysates were incubated with specific antibody or rabbit IgG overnight. Protein G-Sepharose beads were then added for a 2 -h incubation at $4^{\circ} \mathrm{C}$. After centrifugation, the bead pellets were washed with cold $1 \times$ PLB for 15 min four times. In the final wash, $1 \mathrm{M}$ urea was added. RNA was then extracted and purified with RNeasy Plus Micro kit (Qiagen). After reverse transcription with SuperScript III (Invitrogen), qPCR was performed. Gapdh containing a short 3'UTR with no recognizable elements did not bind to the translation regulators, and was used to normalize the data. $\beta$-Actin, Mater, and $Z p 3$ containing no putative CPE were used as negative controls for CPEB1 immunoprecipitation. Eif $4 a 3$ and $Z P 3$ containing no recognizable DAZL-binding element were used as negative controls for DAZL immunoprecipitation. The data are reported as fold enrichment, with IgG values set to 1 .

\section{Reporter mRNA preparation and luciferase assay}

The firefly luciferase (FL) control plasmid was kindly provided by Dr. Yangming Wang (University of California at San Francisco). The renilla luciferase $(\mathrm{RL})$ reporter plasmids were constructed from pRL-TK vector (Promega). The 1841-base-pair (bp) construct for Dazl 3'UTR (1088-2928 of NM_010021.4) was generated by PCR from GV oocyte cDNA using primers AATGAAC 
AATAATTCTCATCTCAGGGGGTTGTTGGTT (forward) and GTGCCACCTGGATCAGGTTTTATTCGTGTATACAAGGA (reverse). The 1630-bp sequence of Tpx2 3'UTR (2723-4352 of NM_028109.4) was generated by PCR from GV oocyte cDNA using primers TGAACAATAATTCTAGAAAGCTGCATCTAT ATCACAAGC and CTTTTTCAATATTATAAACAACATTTA ATTGGGGC. The 440-bp sequence of Tex19.1 3'UTR (12981737 of NM_028602.2) was generated by PCR from GV oocyte cDNA using primers TATCTAGATGCACATTCCTGAGACA CTA and CAAGGATCCAAAACACCAACTTTATTCAACAA AAGC. Constructs were subcloned into pRL-TK downstream from the coding sequence for RL. Three of the four DAZLbinding elements were scrambled by site-directed substitution mutagenesis or deleted in one construct (Tex19.1 Del-RL) All reporters contain a $\mathrm{T} 7$ promoter, allowing in vitro transcription to synthesize mRNAs (Ambion). The FL control mRNA was polyadenylated by Poly(A) Tailing kit (Ambion). Fully grown oocytes were injected with reporter mRNA $(25 \mathrm{ng} / \mu \mathrm{L})$ plus the polyadenylated control FL mRNA and incubated overnight in MEM/BSA containing $2 \mu \mathrm{M}$ Milrinone before culturing in inhibitor-free medium to allow spontaneous maturation. Samples were collected at different times during in vitro maturation. Luciferase assays on the oocyte extracts were carried out using the Dual Luciferase Reporter Assay kit (Promega), and luminescence was detected by SpectraMaxL Luminometer (Molecular Devices). Data are reported as ratio of RL and FL. FL activity did not change significantly in oocytes at different stages of maturation.

\section{$\operatorname{Poly}(A)$ tail assay}

Total RNA isolated from oocytes at indicated stages using RNeasy Plus Micro kit (Qiagen) was hybridized with or without oligo $\mathrm{dT}_{(20)}$ before $\mathrm{RNaseH}$ treatment. RNA was purified and ligated with the anchor primer P1 (5'-P-GGTCACCTTGATCTG AAGC- $\mathrm{NH}_{2}-3^{\prime}$ ) for $1 \mathrm{~h}$ at $37^{\circ} \mathrm{C}$ using T4 RNA ligase (New England Laboratories). Reverse transcription was performed using SuperScript III kit (Invitrogen) with P1 antisense primer P1' (GCTTCAGATCAAG GTGACCTTTTT). The products were used in a PCR reaction with gene-specific primers for Ccnb1 (CCACTCCTGTCTTGTAATGC) or Dazl (GAGAAG GGA GAAAGAGACAAG) and $\mathrm{P} 1^{\prime}$ primer under the following conditions: $30 \mathrm{sec}$ at $94^{\circ} \mathrm{C}, 60 \mathrm{sec}$ at $56^{\circ} \mathrm{C}$, and $60 \mathrm{sec}$ at $72^{\circ} \mathrm{C}$. PCR products were analyzed on a $2.5 \%$ agarose gel.

\section{Statistical analyses}

Experiments were repeated at least three times unless stated. Data are expressed as the mean \pm SEM. Statistical analysis was performed using ANOVA, followed by Bonferroni post-test for comparisons of multiple groups. For comparison between two groups, two-tailed paired $t$-test was used. $P<0.05$ were denoted by one asterisk, $P<0.01$ were denoted by two asterisks, and $P<0.001$ were denoted by three asterisks in Figures $4-7$.

\section{Acknowledgments}

We thank Susan Fisher, Linda Giudice, Miguel Ramalho Santos, Todd Nystul, and Diana Laird for critical reading of the manuscript, and Andrej Susor, Maria Paola Paronetto, and Simona Torcia for technical advice. M.C. is supported by NIH grants RO1-GM080527, RO1HD052909, and U54-HD055764. R.B. is supported by the NIH (K08 NS48118, U54-D055764, and R01NS057221), California Institute of Regenerative Medicine (CIRM) (New Faculty Award RN2-00906), and the Pew Charitable Trust. C.S. is supported by Telethon-Italy.

\section{References}

Arava Y, Wang Y, Storey ID, Liu CL, Brown PO, Herschlag D. 2003. Genome-wide analysis of mRNA translation profiles in Saccharomyces cerevisiae. Proc Natl Acad Sci 100: 38893894.

Arumugam K, Wang Y, Hardy LL, Macnicol MC, Macnicol AM. 2009. Enforcing temporal control of maternal mRNA translation during oocyte cell-cycle progression. EMBO I 29: 387397.

Bayliss R, Sardon T, Vernos I, Conti E. 2003. Structural basis of Aurora-A activation by TPX2 at the mitotic spindle. Mol Cell 12: 851-862.

Belloc E, Mendez R. 2008. A deadenylation negative feedback mechanism governs meiotic metaphase arrest. Nature 452: 1017-1021.

Belloc E, Pique M, Mendez R. 2008. Sequential waves of polyadenylation and deadenylation define a translation circuit that drives meiotic progression. Biochem Soc Trans 36: 665-670.

Blower MD, Feric E, Weis K, Heald R. 2007. Genome-wide analysis demonstrates conserved localization of messenger RNAs to mitotic microtubules. J Cell Biol 179: 1365-1373.

Brook M, Smith JW, Gray NK. 2009. The DAZL and PABP families: RNA-binding proteins with interrelated roles in translational control in oocytes. Reproduction 137: 595-617.

Brunet S, Dumont J, Lee KW, Kinoshita K, Hikal P, Gruss OJ, Maro B, Verlhac MH. 2008. Meiotic regulation of TPX2 protein levels governs cell cycle progression in mouse oocytes. PLoS ONE 3: e3338. doi: 10.1371/journal.pone.0003338.

Collier B, Gorgoni B, Loveridge C, Cooke HJ, Gray NK. 2005. The DAZL family proteins are PABP-binding proteins that regulate translation in germ cells. EMBO J 24: 2656-2666.

De La Fuente R, Eppig JJ. 2001. Transcriptional activity of the mouse oocyte genome: companion granulosa cells modulate transcription and chromatin remodeling. Dev Biol 229: 224 236.

De La Fuente R, Viveiros MM, Burns KH, Adashi EY, Matzuk MM, Eppig JJ. 2004. Major chromatin remodeling in the germinal vesicle $(\mathrm{GV})$ of mammalian oocytes is dispensable for global transcriptional silencing but required for centromeric heterochromatin function. Dev Biol 275: 447-458.

Eliscovich C, Peset I, Vernos I, Mendez R. 2008. Spindle-localized CPE-mediated translation controls meiotic chromosome segregation. Nat Cell Biol 10: 858-865.

Evsikov AV, Graber JH, Brockman JM, Hampl A, Holbrook AE, Singh P, Eppig JJ, Solter D, Knowles BB. 2006. Cracking the egg: molecular dynamics and evolutionary aspects of the transition from the fully grown oocyte to embryo. Genes Dev 20: 2713-2727.

Eyers PA, Maller JL. 2004. Regulation of Xenopus Aurora A activation by TPX2. J Biol Chem 279: 9008-9015.

Grskovic M, Chaivorapol C, Gaspar-Maia A, Li H, RamalhoSantos M. 2007. Systematic identification of cis-regulatory sequences active in mouse and human embryonic stem cells. PLoS Genet 3: e145. doi: 10.1371/journal.pgen.0030145.

Gruss OJ, Carazo-Salas RE, Schatz CA, Guarguaglini G, Kast J, Wilm M, Le Bot N, Vernos I, Karsenti E, Mattaj IW. 2001. Ran induces spindle assembly by reversing the inhibitory effect of importin $\alpha$ on TPX2 activity. Cell 104: 83-93.

Han Z, Liang CG, Cheng Y, Duan X, Zhong Z, Potireddy S, Moncada C, Merali S, Latham KE. 2010. Oocyte spindle proteomics analysis leading to rescue of chromosome congression defects in cloned embryos. J Proteome Res 9: 6025-6032.

Houston DW, King ML. 2000. A critical role for Xdazl, a germ plasm-localized RNA, in the differentiation of primordial germ cells in Xenopus. Development 127: 447-456. 
Huarte J, Stutz A, O'Connell ML, Gubler P, Belin D, Darrow AL, Strickland S, Vassalli JD. 1992. Transient translational silencing by reversible mRNA deadenylation. Cell 69: 1021-1030.

Igea A, Mendez R. 2010. Meiosis requires a translational positive loop where CPEB1 ensues its replacement by CPEB4. EMBO I 29: 2182-2193.

Karashima T, Sugimoto A, Yamamoto M. 2000. Caenorhabditis elegans homologue of the human azoospermia factor DAZ is required for oogenesis but not for spermatogenesis. Development 127: 1069-1079.

Kimble J, Crittenden SL. 2007. Controls of germline stem cells, entry into meiosis, and the sperm/oocyte decision in Caenorhabditis elegans. Annu Rev Cell Dev Biol 23: 405-433.

King ML, Messitt TJ, Mowry KL. 2005. Putting RNAs in the right place at the right time: RNA localization in the frog oocyte. Biol Cell 97: 19-33.

Latham KE, De la Casa E, Schultz RM. 2000. Analysis of mRNA expression during preimplantation development. Methods Mol Biol 136: 315-331.

Lin Y, Page DC. 2005. Dazl deficiency leads to embryonic arrest of germ cell development in XY C57BL/6 mice. Dev Biol 288: 309-316.

Lin Y, Gill ME, Koubova J, Page DC. 2008. Germ cell-intrinsic and -extrinsic factors govern meiotic initiation in mouse embryos. Science 322: 1685-1687.

Liu H, Aoki F. 2002. Transcriptional activity associated with meiotic competence in fully grown mouse GV oocytes. Zygote 10: 327-332.

Ma J, Flemr M, Stein P, Berninger P, Malik R, Zavolan M, Svoboda P, Schultz RM. 2010. MicroRNA activity is suppressed in mouse oocytes. Curr Biol 20: 265-270.

Maegawa S, Yamashita M, Yasuda K, Inoue K. 2002. Zebrafish DAZ-like protein controls translation via the sequence 'GUUC.' Genes Cells 7: 971-984.

Maines JZ, Wasserman SA. 1999. Post-transcriptional regulation of the meiotic Cdc25 protein Twine by the Dazl orthologue Boule. Nat Cell Biol 1: 171-174.

Mendez R, Barnard D, Richter JD. 2002. Differential mRNA translation and meiotic progression require Cdc2-mediated CPEB destruction. EMBO J 21: 1833-1844.

Oh B, Hwang S, McLaughlin J, Solter D, Knowles BB. 2000. Timely translation during the mouse oocyte-to-embryo transition. Development 127: 3795-3803.

Padmanabhan K, Richter JD. 2006. Regulated Pumilio-2 binding controls RINGO/Spy mRNA translation and CPEB activation. Genes Dev 20: 199-209.

Pique M, Lopez JM, Foissac S, Guigo R, Mendez R. 2008. A combinatorial code for CPE-mediated translational control. Cell 132: 434-448.

Potireddy S, Vassena R, Patel BG, Latham KE. 2006. Analysis of polysomal mRNA populations of mouse oocytes and zygotes: dynamic changes in maternal mRNA utilization and function. Dev Biol 298: 155-166.

Radford HE, Meijer HA, de Moor CH. 2008. Translational control by cytoplasmic polyadenylation in Xenopus oocytes. Biochim Biophys Acta 1779: 217-229.

Reijo R, Lee TY, Salo P, Alagappan R, Brown LG, Rosenberg M, Rozen S, Jaffe T, Straus D, Hovatta O, et al. 1995. Diverse spermatogenic defects in humans caused by $\mathrm{Y}$ chromosome deletions encompassing a novel RNA-binding protein gene. Nat Genet 10: 383-393.

Reis A, Madgwick S, Chang HY, Nabti I, Levasseur M, Jones KT. 2007. Prometaphase APCcdh1 activity prevents non-disjunction in mammalian oocytes. Nat Cell Biol 9: 1192-1198.

Reynolds N, Collier B, Maratou K, Bingham V, Speed RM, Taggart M, Semple CA, Gray NK, Cooke HJ. 2005. Dazl binds in vivo to specific transcripts and can regulate the premeiotic translation of Mvh in germ cells. Hum Mol Genet 14: 3899-3909.

Reynolds N, Collier B, Bingham V, Gray NK, Cooke HJ. 2007. Translation of the synaptonemal complex component Sycp3 is enhanced in vivo by the germ cell specific regulator Dazl. RNA 13: 974-981.

Richter JD. 1991. Translational control during early development. Bioessays 13: 179-183.

Richter JD. 2007. CPEB: a life in translation. Trends Biochem Sci 32: $279-285$.

Ruggiu M, Speed R, Taggart M, McKay SJ, Kilanowski F, Saunders P, Dorin J, Cooke HJ. 1997. The mouse Dazla gene encodes a cytoplasmic protein essential for gametogenesis. Nature 389: 73-77.

Schultz RM. 2002. The molecular foundations of the maternal to zygotic transition in the preimplantation embryo. Hum Reprod Update 8: 323-331.

Setoyama D, Yamashita M, Sagata N. 2007. Mechanism of degradation of CPEB during Xenopus oocyte maturation. Proc Natl Acad Sci 104: 18001-18006.

Seydoux G, Braun RE. 2006. Pathway to totipotency: lessons from germ cells. Cell 127: 891-904.

Su YQ, Sugiura K, Woo Y, Wigglesworth K, Kamdar S, Affourtit J, Eppig JJ. 2007. Selective degradation of transcripts during meiotic maturation of mouse oocytes. Dev Biol 302: 104117.

Suh N, Baehner L, Moltzahn F, Melton C, Shenoy A, Chen J, Blelloch R. 2010. MicroRNA function is globally suppressed in mouse oocytes and early embryos. Curr Biol 20: 271-277.

Takeda Y, Mishima Y, Fujiwara T, Sakamoto H, Inoue K. 2009. DAZL relieves miRNA-mediated repression of germline mRNAs by controlling poly(A) tail length in zebrafish. PLoS ONE 4: e7513. doi: 10.1371/journal.pone.0007513.

Tay J, Richter JD. 2001. Germ cell differentiation and synaptonemal complex formation are disrupted in CPEB knockout mice. Dev Cell 1: 201-213.

Tay J, Hodgman R, Richter JD. 2000. The control of cyclin B1 mRNA translation during mouse oocyte maturation. Dev Biol 221: 1-9.

Venables JP, Ruggiu M, Cooke HJ. 2001. The RNA-binding specificity of the mouse Dazl protein. Nucleic Acids Res 29: 2479-2483.

Vitale AM, Calvert ME, Mallavarapu M, Yurttas P, Perlin J, Herr J, Coonrod S. 2007. Proteomic profiling of murine oocyte maturation. Mol Reprod Dev 74: 608-616.

Wang S, Kou Z, Jing Z, Zhang Y, Guo X, Dong M, Wilmut I, Gao S. 2010. Proteome of mouse oocytes at different developmental stages. Proc Natl Acad Sci 107: 17639-17644.

Wolpert L, Jessell T, Lawrence P, Meyerowitz E, Smith J. 2002. Principles of development. Oxford University Press, New York.

Zeng M, Lu Y, Liao X, Li D, Sun H, Liang S, Zhang S, Ma Y, Yang Z. 2009. DAZL binds to 3'UTR of Tex19.1 mRNAs and regulates Tex19.1 expression. Mol Biol Rep 36: 2399-2403.

Zhang P, Ni X, Guo Y, Guo X, Wang Y, Zhou Z, Huo R, Sha J. 2009. Proteomic-based identification of maternal proteins in mature mouse oocytes. BMC Genomics 10: 348. doi: 10.1186/ 1471-2164-10-348. 


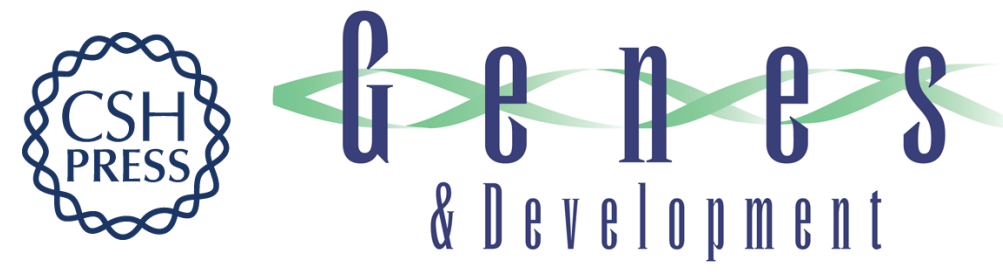

\section{Genome-wide analysis of translation reveals a critical role for deleted in azoospermia-like ( Dazl) at the oocyte-to-zygote transition}

Jing Chen, Collin Melton, Nayoung Suh, et al.

Genes Dev. 2011, 25:

Access the most recent version at doi:10.1101/gad.2028911

Supplemental
Material http://genesdev.cshlp.org/content/suppl/2011/03/30/25.7.755.DC1

References This article cites 56 articles, 14 of which can be accessed free at: http://genesdev.cshlp.org/content/25/7/755.full.html\#ref-list-1

License

Email Alerting

Receive free email alerts when new articles cite this article - sign up in the box at the top Service right corner of the article or click here.

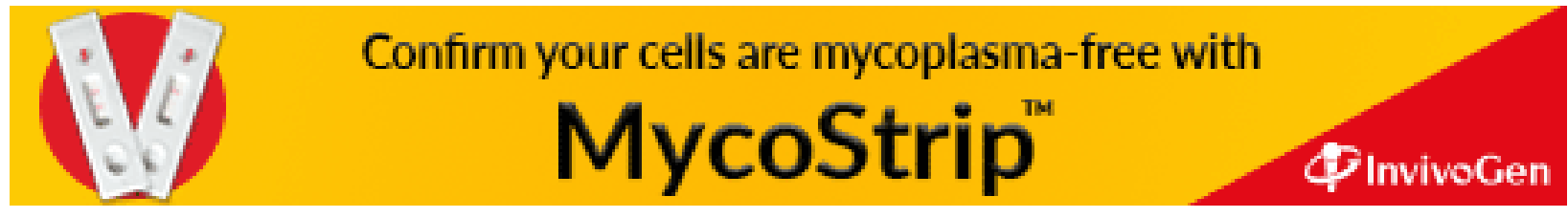

